Revista Saúde.Com

ISSN 1809-0761

www.uesb.br/revista/rsc/ojs

\title{
USO DO PLACEBO EM PESQUISAS CLÍNICAS À LUZ DA BIOÉTICA
}

\section{THE PLACEBO USE IN CLINICAL RESEARCH IN BIOETHICS LIGHT}

Tuany Santos Souza, Silvania Moraes Costa, Edite Lago Da Silva Sena, Sérgio Donha Yarid, Rita Narriman Silva De Oliveira Boery

Universidade Estadual Do Sudoeste Da Bahia - UESB

\begin{abstract}
This study aims to characterize the researches existing in literature about the use of placebos in clinical research. Descriptive research, in the form of integrative literature review by collecting data on the following online bases: BVS, Scopus and Web of Science. Selected articles were analyzed in a summary table, the discussion was based on the Declaration of Helsinki (2008). The use of placebo is questioned under ethical and medical aspects, because it contradicts some bioethical principles such as justice, the beneficence and non-maleficence. It is essential to promote the protection of research participants, before any conflict of interest or appreciation of lucrative eding.
\end{abstract}

Keywords: Placebo. Placebo effect. Bioethics.

\section{Resumo}

Esse estudo objetiva caracterizar as pesquisas existentes na literatura sobre o uso de placebos em pesquisas clínicas. Pesquisa descritiva, sob a forma de revisão integrativa de literatura mediante coleta de dados nas bases on-line BVS, Scopus e Web of Science. Os artigos selecionados foram analisados sob um quadro sinóptico, a discussão fundamentou-se na Declaração de Helsinque (2008). O uso do placebo é questionado sob seus aspectos éticos e médicos, pois contraria alguns princípios bioéticos, como os de justiça, beneficência e não-maleficência. É salutar que se promova a proteção dos participantes da pesquisa, antes de qualquer conflito de interesse ou valorização de fins lucrativos.

Palavras chave: Placebo. Efeito placebo. Bioética. 
Introdução

Ao longo do tempo as pesquisas com seres humanos eram realizadas sem que houvesse uma regulamentação específica, tendo como base a ética do próprio pesquisador, fazendo com que o viés de experimentos abusivos fosse frequente nos registros da história ${ }^{1}$.

Ao final da 2a guerra mundial várias pesquisas rotuladas como crime à humanidade nos campos de concentração, levaram a julgo no Tribunal de Nuremberg (1947) os médicos responsáveis por tais pesquisas, culminando na elaboração do Código de Nuremberg. Foi o primeiro documento criado para tratar sobre ética em pesquisa médica com seres humanos, que se estabeleceu o princípio da autonomia do sujeito de pesquisa ${ }^{2,3,4}$.

Contudo, o Código de Nuremberg não foi o suficiente para impedir a procedência de experimentos abusivos, despertando uma inquietação sobre a questão bioética na condução de tais pesquisas. Assim, em 1964, durante a 18a Assembleia da Associação Médica Mundial na Finlândia, foi aprovado outro documento - Declaração de Helsinki - contendo novas exigências éticas com o intuito de respaldar a segurança e o respeito aos seres humanos participantes de pesquisas clínicas. Essa declaração tem sido amplamente revisada, desde sua criação, sendo sua ultima versão atualizada no ano de $2008^{5}$.

Neste contexto, dentre as modificações propostas pela Declaração de Helsinque, destacam-se a revisão dos projetos de pesquisa pelos comitês de ética, a restrição do uso de placebo e a garantia de continuidade de tratamento aos participantes dos estudos ao término dos mesmos ${ }^{6}$.

Embora originalmente os placebos fossem preparações medicinais sem atividade farmacológica específica contra uma condição alvo, o conceito foi estendido para incluir tratamentos ou procedimentos, visando obter medidas basais para o protocolo experimental. Em outras palavras, os placebos, por sua vez, compreendem qualquer medicamento ou tratamento fictício, para fins de avaliações em ensaios clínicos controlados ${ }^{7}$. Dessa forma, o uso de placebos nas relações profissionais não se trata apenas de um problema metodológicocientífico, é, sobretudo, uma questão bioética e sua utilização na medicina é questionável do ponto de vista da proteção dos sujeitos e da garantia de seus direitos ${ }^{8}$.

Geralmente, o uso dos placebos é justificado pela falta de um fármaco considerado padrão-ouro ou pela desconfiança do pesquisador na avaliação subjetiva que o indivíduo participante da pesquisa fará. Trata-se, portanto, de uma dissimulação terapêutica que a princípio não trará nenhum benefício direto a quem o utilizar ${ }^{4}$. Todavia, existindo tratamento minimamente eficaz para a doença não é eticamente correto deixar um grupo de pacientes sem terapia, sendo que o experimento deveria comparar, então, o novo tratamento com o tratamento existente ou padrão ${ }^{9}$.

Não obstante, questiona-se qual o comportamento da literatura em relação ao uso de placebos em pesquisas clínicas? Nesta perspectiva, o objetivo desse estudo é caracterizar as pesquisas existentes na literatura sobre o uso de placebos em pesquisas clínicas, à luz da Bioética.

\section{Metodologia}

Trata-se de uma pesquisa descritiva, realizada sob a forma de revisão integrativa de literatura, cujo método se propõe a reunir e sintetizar resultados de pesquisas sobre um determinado tema, de forma sistemática e ordenada, contribuindo para o aprofundamento do entendimento sobre o mesmo ${ }^{10}$.

As etapas desse estudo foram sistematizadas na identificação do tema, delineamento de critérios para inclusão e exclusão dos estudos, categorização e avaliação dos estudos incluídos na revisão integrativa, interpretação dos resultados e apresentação da revisão/síntese do conhecimento.

Para tanto, o levantamento foi realizada mediante consulta às bases de dados on-line Biblioteca Virtual em Saúde (BVS), Scopus e Web of Science, no período de abril a maio de 2015, através dos descritores "placebo", "efeito placebo", "bioética", com interrelação dos operadores booleanos and e or. Utilizou-se também a Declaração de Helsinki (2008) para fundamentação da discussão.

Os critérios de inclusão considerados foram: artigos completos originais ou de revisão disponíveis gratuitamente, que abordassem como tema principal Placebo e Bioética. Foram encontrados inicialmente 13 artigos disponíveis no banco de dados da Biblioteca Virtual de Saúde - BVS, 4 na Scopus e 4 na Web of Science. Realizou-se uma leitura flutuante dos títulos dos estudos encontrados, excluindo-se aqueles que apresentaram duplicidade entre as bases, selecionando-se 12 artigos. Estes, por sua vez, passaram por leitura exaustiva de todo o corpo do texto, a fim de selecionar apenas os que de 
fato abordavam a temática pesquisada. Sendo assim, a amostra final foi composta por seis artigos que atendiam aos objetivos e critérios de inclusão, com recorte temporal de 2007 à 2015.

Por fim, foi realizada a caracterização dos estudos por meio de fichamento e compilação de algumas variáveis observadas: referência do artigo (título, identificação dos autores e do periódico, local e ano de publicação), tipo de estudo e os principais resultados e conclusões.

\section{Resultados e Discussões}

Os estudos selecionados são artigos de pesquisa originais, reflexões teóricas e artigos de revisão. Estes estudos foram categorizados e suas informações estão apresentadas no Quadro 1.

Quadro 1. Artigos selecionados e utilizados na revisão. Jequié, Bahia, Brasil, 2015.

\begin{tabular}{|c|c|c|}
\hline REFERÊNCIA DO ARTIGO & TIPO DE ESTUDO & PRINCIPAIS RESULTADOS E CONCLUSÕES \\
\hline $\begin{array}{l}\text { Garrafa V, Lorenzo C. Helsinque 2008: } \\
\text { redução de proteção e maximização de } \\
\text { interesses privados. Rev. Assoc. Med } \\
\text { Bras. 2009; 55(5): 497-520. }\end{array}$ & Reflexão Teórica & $\begin{array}{l}\text { Nos últimos anos, é muito provável que as } \\
\text { modificações feitas na Declaração de } \\
\text { Helsinque reduziram a proteção de sujeitos e } \\
\text { comunidades e maximizaram os interesses das } \\
\text { grandes indústrias farmacêuticas. Normas } \\
\text { guias internacionais são indispensáveis para } \\
\text { dar o rumo a ser seguido no desenvolvimento } \\
\text { das pesquisas clínicas em cada país do planeta. } \\
\text { Mas as particularidades e necessidades de } \\
\text { cada nação devem - definitivamente - ser } \\
\text { consideradas para que os ensaios clínicos de } \\
\text { métodos diagnósticos, preventivos ou } \\
\text { terapêuticos, enquanto atividades sociais } \\
\text { globalizadas realizem-se de forma a respeitar } \\
\text { os direitos humanos fundamentais. }\end{array}$ \\
\hline $\begin{array}{l}\text { Miranda E,Hossne WS. Ensaios clínicos } \\
\text { (fase III) - perfil das publicações } \\
\text { científicas. Aspectos bioéticos. Ver Bras } \\
\text { Clin Med. 2010; } 8(2): 143-7 \text {. }\end{array}$ & Artigo de Revisão & $\begin{array}{l}\text { Com base na análise de } 96 \text { publicações } \\
\text { referentes à pesquisa clínica fase III, tomando } \\
\text { como referencial as diretrizes éticas atinentes } \\
\text { à pesquisa envolvendo seres humanos e os } \\
\text { documentos regulatórios técnico-científicos, os } \\
\text { autores concluíram que a publicações de fase } \\
\text { III deixam de fornecer ao leitor e, em ultima } \\
\text { análise, à comunidade científica, elementos } \\
\text { suficientes para a sua própria avaliação ética e } \\
\text { tecnocientifica. }\end{array}$ \\
\hline $\begin{array}{l}\text { Rubenich GB, Heck ST, Hellmann F, } \\
\text { Schlemper Junior BR. El uso de placebo } \\
\text { em ensayos clínicos de fase III em } \\
\text { Brasil. Salud Colectiva. 2015; 11(1):99- } \\
\text { 114. }\end{array}$ & Artigo de Revisão & $\begin{array}{l}\text { O Conselho Federal de Medicina do Brasi } \\
\text { (CFM) proibiu em 2008, a participação dos } \\
\text { médicos Brasileiros em investigações que } \\
\text { utilizarão placebo para enfermidades com } \\
\text { tratamento eficiente e eficaz, em } \\
\text { contraposição à Declaração de Helsinque, que } \\
\text { permite a sua utilização em condições } \\
\text { metodologicamente justificadas. Conclui-se } \\
\text { que: a) os regulamentos emitidos pelo CFM em } \\
\text { 2008, foram ineficazes e prevaleceu a posição } \\
\text { assumida pela Declaração de Helsinque; b) O } \\
\text { patrocínio de ensaios com placebo por parte } \\
\text { da indústria farmacêutica multinacional fo } \\
\text { significativo; c) predominaram pesquisas sobre } \\
\text { fármacos para doenças crônicas, e eram } \\
\text { insignificantes para as doenças negligenciadas, } \\
\text { de importância para o Brasil. }\end{array}$ \\
\hline
\end{tabular}

Continua... 


\begin{tabular}{|c|c|c|}
\hline $\begin{array}{l}\text { Sousa MAS, Franco MAG, Filho JM. A } \\
\text { nova declaração de Helsinque e o uso } \\
\text { de placebo em estudos clínicos no } \\
\text { Brasil: a polêmica continua. Rev Med. } \\
\text { 2012; 91(3):178-88. }\end{array}$ & Artigo de Revisão & $\begin{array}{l}\text { Apesar da divergência dentre vários grupos, } \\
\text { sobretudo, quanto ao uso do placebo, o texto } \\
\text { atual assumiu uma posição neutra, } \\
\text { flexibilizando sua aplicação. Todavia, uma vez } \\
\text { norma ética, a atual Declaração de Helsinque } \\
\text { fomentou o reinício das discussões sobre a } \\
\text { necessidade de um documento mais amplo e } \\
\text { uniformemente aceito. Esta polêmica ainda } \\
\text { continua em alguns países, principalmente no } \\
\text { Brasil, onde o Conselho Federal de Medicina } \\
\text { (CFM) e a Comissão Nacional de Ética em } \\
\text { Pesquisa (CONEP) proíbem o uso de placebo } \\
\text { em estudos clínicos em território nacional, } \\
\text { quando existir qualquer tratamento disponível. } \\
\text { Este fato eliciou grande polêmica entre } \\
\text { agências regulatórias e pesquisadores } \\
\text { brasileiros. }\end{array}$ \\
\hline $\begin{array}{l}\text { Teixeira M. Placebo, um mal-estar para } \\
\text { a medicina: notícias recentes. Rev. } \\
\text { Latinoam. Psicopat. Fund. 2008; } \\
\text { 11(4):653-60. }\end{array}$ & Reflexão Teórica & $\begin{array}{l}\text { Uma linha de investigação mais difundida a } \\
\text { propósito do placebo e seus efeitos, no } \\
\text { enquadre da medicina científica, é aquela que } \\
\text { pretende saber sobre a ética de seu uso. Em } \\
\text { outubro de 2008, a Associação Médica } \\
\text { Mundial, reunida em assembléia em Paris, } \\
\text { mudou o texto do artigo } 32 \text { da Declaração de } \\
\text { Helsinque, que regula a participação de seres } \\
\text { humanos na pesquisa clínica. A norma vigente } \\
\text { até a assembléia de outubro vetava a } \\
\text { possibilidade do uso de placebo em ensaios } \\
\text { clínicos, quando há outro tratamento } \\
\text { disponível. No novo texto do artigo } 32 \text {, "o uso } \\
\text { de placebo, ou o não-tratamento" recebeu a } \\
\text { qualidade de "aceitável" quando, para a } \\
\text { doença estudada, não existir current provenint } \\
\text { ervention. A idéia de que o ser humano } \\
\text { envolvido em um experimento deve ser } \\
\text { protegido do uso do placebo baseia-se na } \\
\text { concepção do placebo como "substância } \\
\text { inócua". É porque o placebo é "inócuo" que, } \\
\text { para a bioética, não se pode utilizá-lo quando } \\
\text { há outro tratamento disponível - seria como } \\
\text { negar socorro, o que contraria a exigência de } \\
\text { que a participação em um ensaio clínico não } \\
\text { traga dano ao paciente. }\end{array}$ \\
\hline $\begin{array}{l}\text { Garrafa V, Prado MM. Alterações na } \\
\text { Declaração de Helsinque - a história } \\
\text { continua. Revista Bioética. 2007; } \\
\text { 15(1):11-25. }\end{array}$ & Reflexão Teórica & $\begin{array}{l}\text { Os autores analisaram o papel histórico } \\
\text { representado pela Declaração de Helsinque no } \\
\text { contexto da ética da investigação com seres } \\
\text { humanos e observaram que, principalmente, } \\
\text { os artigos } 19,29 \text { e } 30 \text { do documento tiveram } \\
\text { sua legitimidade questionada. Esses artigos } \\
\text { referem-se à utilização de double standard } \\
\text { (padrão duplo) nas investigações que refere-se } \\
\text { ao não cumprimento da ética e sem obrigação, } \\
\text { por parte dos patrocinadores, em continuar os } \\
\text { cuidados aos sujeitos, após o estudo ter sido } \\
\text { concluído. Este artigo faz uma análise do longo } \\
\text { processo de tentativas de alterações criticando } \\
\text { duramente sob a luz da bioética, as posições } \\
\text { imperialistas e unilaterais dos americanos. }\end{array}$ \\
\hline
\end{tabular}

Fonte: Dados da pesquisa e interpretação dos autores 
Nos estudos analisados discute-se sobre a incoerência na condução de algumas pesquisas clínicas que com maior frequência são consideradas eticamente incorretas, especialmente aquelas que se referem ao uso do placebo-controle para fins de investigação da eficácia de novos fármacos.

É pequeno o número de laboratórios farmacêuticos que se propõem a conduzir pesquisas com 0 objetivo de produzir medicamentos que atendam às necessidades epidemiológicas de países mais pobres, pois os ganhos financeiros não compensam, uma vez que nesta ceara o conflito de interesses entre indústria farmacêutica e pesquisadores médicos é muito grande ${ }^{11}$.

Por outro lado, as pesquisas podem ser consideradas 'cooperativas' desde que haja acordos bilaterais entre as instituições que fomentam a pesquisa e os países-sede, tendo em vista que a pesquisa busque métodos terapêuticos preventivos ou diagnósticos relacionados à resolução dos problemas de saúde da população participante, bem como, contribuam para o desenvolvimento da população mediante transferência de tecnologias, práticas avançadas de investigação, dentre outros benefícios ${ }^{8}$.

Entretanto, existe a possibilidade de que as pesquisas internacionais sejam realizadas sobre o cunho de características de exploração sobre os sujeitos sempre que a participação dos países periféricos vise a tão somente burlar uma supervisão ética mais rigorosa, alocar sujeitos com desvantagens econômicas a fim de acelerar as etapas de recrutamento, assim como, não disponibilizar os benefícios gerados no final do estudo aos grupos sociais que participaram do mesmo ${ }^{12}$.

Neste contexto, os estudos analisados nesta revisão põem em questionamento o valor metodológico do placebo sob seus aspectos éticos e médicos, já que contraria os princípios fundamentais da bioética: o de justiça, pois todos os participantes devem ter o direito de acesso ao melhor tratamento possível, com igual razão benefício/risco; o de beneficência, uma vez que não se deve causar danos e deve-se ampliar o número de possíveis benefícios, reduzindo riscos; e o de não-maleficência, já que não é aceitável a possibilidade de um voluntário, de modo previsto, vir a ser lesado no ensaio clínico ${ }^{13,14}$.

Por esta razão, existem no meio científico alguns argumentos de cunho ético-médico contrários ao uso do placebo, embasados no fato de que os placebos são farmacologicamente inertes, logo, é repreensível seu uso apenas para fins estatísticos, além do que, atualmente, já estão mais facilmente disponíveis tratamentos alternativos que possam ser empregados como controle ativo nos ensaios clínicos ${ }^{14}$.

Além disso, Oliveira (2006) destaca que de acordo com a própria Declaração de Helsinki, é inaceitável que um grupo de pessoas submetidas a ensaios controlados com placebo seja colocado em risco de subtratamento para o benefício teórico, em potencial, de um grupo hipotético de pacientes, ou mesmo da população em geral ${ }^{15}$.

Essas não conformidades é que impulsionam a criação de documentos em prol da proteção de sujeitos e grupos sociais vulneráveis a esses dilemas éticos, como a Declaração de Helsinki, que se trata do documento mundial mais abrangente sobre ética em pesquisa com seres humanos ${ }^{13}$. A versão atual da Declaração de Helsinki (2008) passou a ser a única versão oficial, substituindo as anteriores e trazendo consigo muitos debates referentes às alterações inerentes à pesquisa clínica ${ }^{6}$.

Segundo Garrafa e Lourenzo (2009) as modificações na Declaração de Helsinki, especialmente nos seus antigos tópicos 19, 29 e 30 , referentes á regulação ética do uso do placebo e ao acesso de benefícios ao fim do estudo, maximizam os interesses das grandes multinacionais indústrias farmacêuticas, enquanto ferem a proteção dos sujeitos e comunidades socialmente vulneráveis nos países subdesenvolvidos ${ }^{6,8}$.

Do ponto de vista da bioética o significado da vulnerabilidade social conduz ao contexto de fragilidade, desproteção, desfavorecimento ou mesmo abandono e desamparo. Tratando-se das pesquisas clínicas, seriam as situações em que se pode interferir na autonomia dos sujeitos em participar ou não das pesquisas; riscos excedentes provenientes da pesquisa ou qualquer prejuízo à capacidade de defesa dos próprios interesses em relação aos benefícios gerados $^{15}$.

Segundo alguns bioeticistas, as mudanças na Declaração de Helsinki devem ser repensadas e postas à debate, já que elas dão margem a existência de pesquisas médicas que visam na maioria das vezes o lucro, demonstrando que as considerações éticas são irrelevantes quando se trata de países pobres ${ }^{16,17}$.

\section{Conclusão}

Diante da reflexão bioética acerca do uso do placebo nas pesquisas clínicas com seres 
humanos, percebe-se que esta temática ainda precisa ser discutida no meio científico, visando a beneficência dos sujeitos submetidos a tais estudos. Entretanto, poucos artigos abordam essa temática, sugerindo que mais estudos possam ser elaborados para difundir este campo de discussões, a fim de divulgar no meio científico a relevância dessas reflexões.

Não cabe apenas à Associação Médica Mundial ou aos Comitês de Ética em Pesquisas decidir de forma unilateral por todas as populações e sociedades do planeta sobre assunto tão complexo e plural. A decisão pela utilização ou não do placebo nos ensaios controlados para fins de comparação e testes de eficácia requer um pensar coletivo, uma soma de esforços e, sobretudo um controle social. Frente a isso, a necessidade de um padrão de conduta conciso e uniforme se faz cada vez mais emergente.

Vale, portanto, um olhar coletivo, social e humano para que esse dilema bioético não se torne invisível ao ponto de não causar incômodo. Pelo contrário, o uso do placebo deve ser criteriosamente avaliado, caso a caso, para que os riscos de se participar de um estudo clínico não venham a sobrepujar os possíveis benefícios e, sobretudo, que seja promovido aquilo que está disposto nos documentos oficiais, como a Declaração de Helsinki: a proteção dos participantes da pesquisa como objetivo primordial.

\section{Referências}

1. Miranda E, Hossne WS. Ensaios clínicos (fase III) - perfil das publicações científicas. Aspectos bioéticos. Rev Bras Clin Med. 2010; 8(2): 143-7.

2. Shuster E. Fifty years later: The significance of the Nuremberg Code. $N$ Engl J Med.1997; 337(20): 1436-40.

3. Castilho EA, Kalil J. Ética e pesquisa médica: princípios, diretrizes e regulamentações. Rev. Soc. Bras. Med. Trop.2005; 38(4).

4. Greco DB, Parizi R. Bioética e uso de placebo em pesquisas clínicas. Brasília Med. 2014; 51(1): 1-3.

5. Sousa VD. Regulação técnica e bioética da participação de seres humanos em ensaios clínicos de bioequivalência. Dissertação. Universidade de Brasilia - DF. 2010.

6. World Medical Association. WMA Declaration of Helsinki - Ethical Principles for Medical Research Involving Human Subjects, Fortaleza, Brazil 2013.
7. Shapiro AK, Shapiro E. The powerful placebo: from acient priest to modern physician. Baltimore: John's Hopkins University Press; 1997.

8. Garrafa V, Lorenzo C. Helsinque 2008: redução de proteção e maximização de interesses privados. Rev Assoc Med Bras. 2009; 55(5): 497-520.

9. Costa SIF, Garrafa V, Oselka G. Apresentando a Bioética. In: Costa SIF, Garrafa V, Oselka G, organizadores. Iniciação à bioética. Brasília: Conselho Federal de Medicina; 1998: 1518.

10. Mendes KD, Silveira RCCP, Galvão CM. Revisão integrativa: método de pesquisa para a incorporação de evidências na saúde e na enfermagem. Texto Contexto enferm, Florianópolis. 2008; 17(4); 758-764.

11. Angell MA. Verdade sobre os laboratórios farmacêuticos. 3a ed. Rio de Janeiro: Record; 2007.

12. Teixeira M. Placebo, um mal-estar para a medicina: notícias recentes. Rev. Latinoam. Psicopat. Fund., São Paulo. 2008; 11(4): 653-660.

13. Sousa MAS, Franco MAG, Filho JM. A nova declaração de Helsinque e o uso de placebo em estudos clínicos no Brasil: a polêmica continua. Rev Med, São Paulo. 2012; 91(3): 17888.

14. Rubenich GB, Heck ST, Hellmann F, Schlemper Junior BR. El uso de placebo en ensayos clínicos de fase III em Brasil. Salud Colectiva, Buenos Aires. 2015; 11(1): 99-114.

15. Oliveira GG. Ensaios clínicos: princípios e prática. Brasília: Ministério da Saúde, Agência Nacional de Vigilância Sanitária; 2006.

16. Garrafa V, Lorenzo C. Imperialismo moral e ensaios clínicos multicêntricos em países periféricos. Cad Saúde Pública. 2008; 24(10): 2219-26.

17. Lurie P, Greco DB. Us exceptionalism comes to research ethics. Lancet, v. 2005; 365(9465): 1117-9.

\section{Endereço para Correspondência}

Universidade Estadual do Sudoeste da Bahia UESB

Rua José Moreira Sobrinho, s/n Campus de Jequiezinho, Jequié - BA

CEP.: 45205-490

e-mail: silvaniamoraescosta@hotmail.com

Recebido em 26/03/2018

Aprovado em 06/09/2018

Publicado em13/03/2019 